\title{
Sequential compression pump effect on hypotension due to spinal anesthesia for cesarean section: A double blind clinical trial
}

Fatemeh Javaherforoosh Zadeh ${ }^{1}$, Mostafa Alqozat ${ }^{2}$, Reza Akhond Zadeh ${ }^{1}$

\begin{abstract}
${ }^{1}$ M.D., Anesthesiologist, Associate Professor, Department of Anesthesia, Pain Research Center, Ahvaz Jundishapur University of Medical Sciences, Ahvaz, Iran

${ }^{2}$ M.D., Gynecologist, Assistant Professor, Fertility Infertility and Perinatology Research Center, Ahvaz Jundishapur University of Medical Sciences, Ahvaz, Iran
\end{abstract}

Type of article: Original

\begin{abstract}
Background: Spinal anesthesia (SA) is a standard technique for cesarean section. Hypotension presents an incident of $80-85 \%$ after SA in pregnant women.

Objective: To determine the effect of intermittent pneumatic compression of lower limbs on declining spinal anesthesia induced hypotension during cesarean section.

Methods: This double-blind clinical prospective study was conducted on 76 non-laboring parturient patients, aged 18-45 years, with the American Society of Anesthesiologist physical status I or II who were scheduled for elective cesarean section at Razi Hospital, Ahvaz, Iran from December 21, 2015 to January 20, 2016. Patients were divided into treatment mechanical pump (Group M) or control group (Group C) with simple random sampling. Fetal presentation, birth weight, Apgar at 1 and $5 \mathrm{~min}$, time taken for pre-hydration (min), prehydration to the administration of spinal anesthesia ( $\mathrm{min}$ ), initiation of spinal to the delivery (min) and total volume of intravenous fluids, total dose of ephedrine and metoclopramide were recorded. Data were analyzed by SPSS version 19, using repeated measures of ANOVA and Chi square test.

Results: Heart rate, MPA, DAP and SAP changes were significantly higher in off-pump group in the baseline and 1 st-minute $(\mathrm{p}<0.05)$, and in the other times, this change was significantly different with control groups.

Conclusion: This research showed the suitability of the use of Sequential Compression Device (SCD) in reducing hypotension after spinal anesthesia for cesarean section, also this method can cause reducing vasopressor dosage for increased blood pressure, but the approval of its effectiveness requires repetition of the study with a larger sample size.

Trial registration: The trial was registered at the Iranian Registry of Clinical Trials (http://www.irct.ir) with the IRCT ID: IRCT2015011217742N3.

Funding: The authors received no financial support for the research, authorship, and/or publication of this article.

Keywords: Sequential Compression Pump, Hypotension, Cesarean Section, Spinal Anesthesia
\end{abstract}

\section{Introduction}

Spinal anesthesia (SA) is a standard technique for cesarean section $(1,2)$. Hypotension is defined by a systolic pressure drop by more than $20 \%$ of the baseline, or a pressure drop by more than $90-100 \mathrm{~mm} / \mathrm{Hg}$. This complication presents an incident of $80-85 \%$ after SA in pregnant women (3). The hypotension is related to venous pooling secondary to progesterone-induced decrease in vascular tone, and is dangerous to mother, fetus, and newborn (4). The rigorousness of hypotension is dependent on the altitude of the block, the arrangement of the patient, the volume, and type of surgery. The procedures that decrease hypotension consist of volume loading, inotropic agents, prevention of aortocaval compression, and regular blood pressure monitoring. Early detection and management of hypotension can significantly reduce the risk to mother and fetus. Hypotension due to SA is caused by an increase in

\section{Corresponding author:}

Associate Professor Dr. Fatemeh Javaherforoosh Zadeh, Department of Anesthesia, Pain Research Center, Ahvaz Jundishapur University of Medical Sciences, Ahvaz, Iran.

Tel: +989161114831, Fax: +986132923980, Email:f javaherforoosh@yahoo.com

Received: May 02, 2016, Accepted: September 22, 2016, Published: May 2017

iThenticate screening: September 17, 2016, English editing: April 12, 2017, Quality control: May 02, 2017

(C) 2017 The Authors. This is an open access article under the terms of the Creative Commons Attribution-NonCommercialNoDerivs License, which permits use and distribution in any medium, provided the original work is properly cited, the use is non-commercial and no modifications or adaptations are made. 
venous pooling as a consequence of parasympathetic over-activation, causing vasodilation in the lower part of the body. This situation is more severe in pregnant women, due to aortocaval compression. Under normal conditions, hypotension in pregnant women is compensated by increased cardiac output; however, after SA-induced sympathectomy, this compensation is attenuated or sometimes eliminated, and thus leads to a heart rate drop, followed by a cardiac output decline (6). Since uterine blood flow is pressure-dependent (due to the lack of a placental auto-regulation), prolonged maternal hypotension is harmful to the fetus (due to lower Apgar scores and fetal acidosis). It is also frequently associated with nausea and vomiting for the mother (7). As stated earlier, there are several methods for decreasing SA-induced hypotension, including the use of Sequential Compression Device (SCD). In this method, the pressure is intermittently put above the knee. According to previous studies, almost 125 cc of blood is moved in compression phase. Although there is no comparative study into the blood volume among pregnant and non-pregnant women, it is well known that there is a high blood volume in the lower extremities at term, which is further increased by SA through vasodilatation (8). Therefore, theoretically, the SCD method mobilizes more blood to the central [active] blood volume, and thus decreases hypotension in a more stable form; whereas, other methods are relatively short-term and temporary. Due to the limited amount of relevant research, this study intended to determine the effect of intermittent pneumatic compression of lower limbs on declining SAinduced hypotension during $\mathrm{C}$-section.

\section{Material and Methods}

\subsection{Trial design and participants}

This study was a randomized clinical trial that was conducted from December 21, 2015 to January 20, 2016 in a governmental educational hospital (Razi Hospital, Ahwaz, Iran).

\subsection{Selection criteria}

The inclusion criteria for participation in this study were: 1) non-laboring parturient, 2) anesthesiologist physical status I or II, 3) elective cesarean section, and 4) age between 18-45 years. The exclusion criteria included:1) patients in whom spinal anesthesia was contraindicated, 2) emergency cesarean section, 3) multiple gestation, 4) pregnancy pathology, 5) heart diseases, 6) history of hypertension or pregnancy-induced hypertension ,7) body mass index (BMI) $>40$, and 8) gestational diabetes.

\subsection{Interventions}

This double-blind clinical prospective study was conducted on 76 non-laboring parturient patients. After approval of the ethics committee of Ahvaz Jundishapur University of Medical Sciences and obtaining written informed consent, patients were divided in to treatment mechanical pump (Group M) or control group (Group C) with simple random sampling. The following variables were noted: body mass index, gestational age and medical history. In the operating room, all patients were situated at 15 degrees left lateral position and routine monitoring was applied for continuous monitoring. Baseline systolic, diastolic and mean arterial pressures (SAP, DBP and MAP) and heart rate (HR) were recorded and then pre-hydration with $10 \mathrm{ml} / \mathrm{kg}$ lactated ringer solution was administrated. Two groups had appropriate sized sleeves applied to the lower limbs. Discontinuous compression started with a pressure of 50 $\mathrm{mmHg}$ in $\mathrm{M}$ group patients. Spinal anesthesia was performed in the sitting position using a $25 \mathrm{G}$ Sprotte needle in the L3-4 or L4-5 interspace. All patients received $10 \mathrm{mg}$ of bupivacaine $0.5 \%$. Subsequently, the patients were positioned supine with a 15-degree left lateral angle. Five minutes after SA, the upper sensory level of anesthesia was measured. The arterial blood pressure was measured and recorded each minute until delivery and thereafter, every 3 minutes until 10 minutes, and then every 5 minutes until arrival to recovery room, and finally, every 10 minutes until discharge from recovery room. Hypotension was defined either as a decrease in any MAP measurement to less than $20 \%$ of the base line, or SAP less than $90 \mathrm{mmHg}$. If Hypotension occurred, it was treated by an anesthesia nurse, who was blinded to the protocol, with $5 \mathrm{mg}$ increments of intravenous ephedrine boluses until corrected and intravenous fluid administration simultaneously.

\subsection{Outcomes}

The primary outcome of the study was defined as the incidence of hypotension. Secondary outcomes that we compared, included serial changes in blood pressure, nausea or vomiting, HR, and Apgar scores at 1 and 5 min. Nausea and vomiting were treated with metoclopramide. Total dose of ephedrine and metoclopramide was recorded

\subsection{Sample size}

The sample size was calculated based on the results of previous studies $(1,9)$ by assuming the test power of $80 \%$ and a confidence level of $95 \%$ and using the following formula: $\mathrm{n}=\left(\mathrm{Z}_{1-\alpha / 2}+\mathrm{Z}_{1-\beta}\right)^{2} \times\left(\left(\mathrm{P}_{1} \times\left(1-\mathrm{P}_{1}\right)+\mathrm{P}_{2} \times\left(1-\mathrm{P}_{2}\right)\right) /\left(\mathrm{P}_{1}-\mathrm{P}_{2}\right)^{2}\right.$; 
Where: $\mathrm{n}=$ Sample size, $\mathrm{Z}_{1-\alpha / 2}=1.96$ when $\alpha=5 \%$ for two-tailed hypothesis, $Z_{1-\beta}=0.842$ when $\beta=20 \%$ (test power $=80 \%), \mathrm{P}=$ Probability of the main outcome.

\subsection{Randomization and blinding}

Patients were divided into treatment mechanical pump (Group M) or control group (Group C) with simple random sampling. Then, the participants in each of the groups were assigned randomly to receive either mechanical pump or control on a 1:1 ratio. Randomization was done by one of the researchers who did not have a role in the treatment of the participants.

\subsection{Statistical methods}

Statistical analyses were performed using SPSS version 19 (SPSS Inc., Chicago, Illinois, USA). The results are expressed as mean $\pm(\mathrm{SD})$, median (range) or number (\%). $\mathrm{p}<0.05$ was considered as significant. The comparison of normally distributed continuous variables between the groups was performed using students' $T$ test. Nominal categorical data between the groups were compared using the Chi square. Serial data were analyzed by repeated measures of ANOVA. The incidence of hypotension was compared using the Chi square test.

\subsection{Research ethics.}

This research was approved by the Pain Research Center, Ahvaz Jundishapur University of Medical Sciences, Ahvaz, Iran (Proposal No. PAIN-9312). In this study, for ethical considerations, the participants were informed about the objective and nature of the study, and each participant provided her written consent in her native language (Persian) prior to the study. Also, we were committed to keeping all of the participants' information confidential.

\section{Results}

Seventy-six patients were randomly assigned between two groups $\mathrm{M}$ and $\mathrm{C}$. Three patients in group $\mathrm{M}$ were excluded (one patient because of inadequate spinal block and two patients because severe shivering prevented accurate measurement of blood pressure). Patient characteristics and surgical times were similar between two groups (Table 1). There is no difference in hypotension between the two groups $(p=0.17)$. The MAP changes were significantly higher in the $\mathrm{C}$ group only at the baseline $(\mathrm{p}<0.05)$. In addition, the lowest MAP belonged to the $\mathrm{C}$ group in the 35th-minute, and the highest MAP belonged at the baseline in this group. These changes were not statistically significant (Figure1).

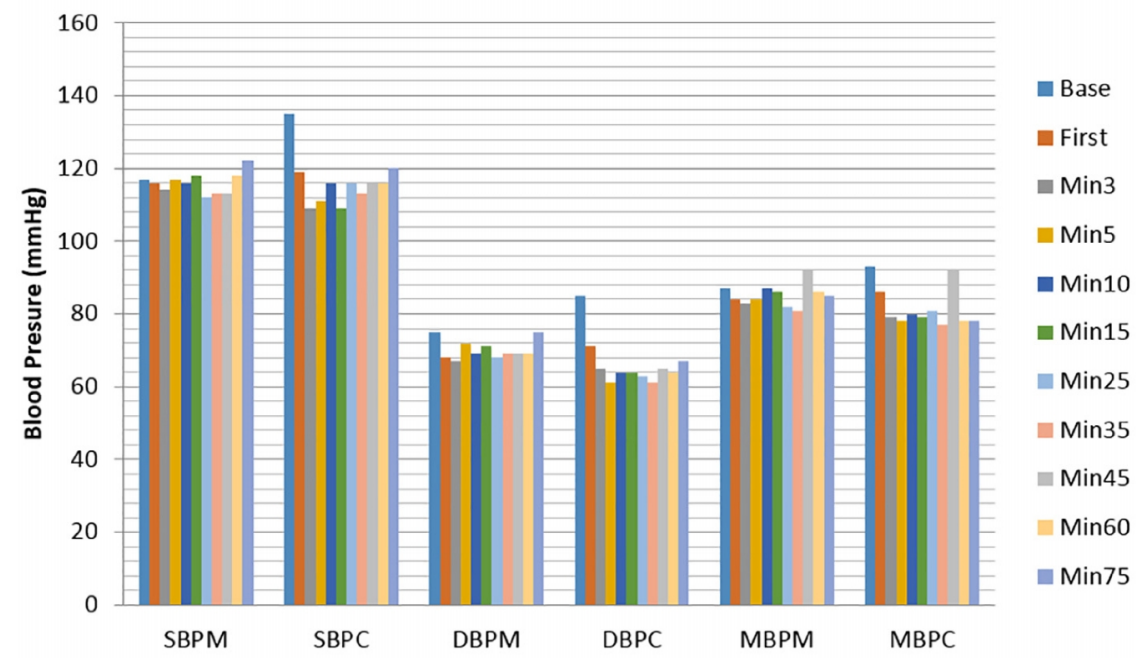

Figure 1. Serial changes in systolic blood pressure, diastolic blood pressure and mean blood pressure. Data are presented as mean \pm SD. SBP: systolic blood pressure, DBP: diastolic blood pressure, MBP: Mean blood pressure. $\mathrm{M}$ and $\mathrm{C}$ denote treatment mechanical pump group and control group respectively.

The SAP changes were significantly higher in the $\mathrm{C}$ group only at the baseline, but at other times, with no significant difference between two groups. In addition, the lowest SAP belonged to the $\mathrm{C}$ group in the 3rd- and 15thminute, and the highest SAP belonged to the $\mathrm{C}$ group at the baseline. These changes were not significant. DAP changes were significantly higher in the $\mathrm{C}$ group in the baseline and 1 st-minute $(\mathrm{p}<0.05)$. These changes were significantly higher in the M group in the 5th-minute. In addition, the lowest DAP belonged to the $\mathrm{C}$ group in the 
5th- and 35th-minute, and the highest DAP belonged to the $\mathrm{C}$ group at the baseline. These changes were not statistically significant (Figure 1, Table 2). Heart rate changes were significantly higher in the $\mathrm{M}$ group in the first and third minutes $(p<0.05)$. In addition, the lowest heart rate belonged to the $C$ group in the third-minute, and the highest heart rate belonged to the $M$ group in the 15 th-minute. These changes were not statistically significant (Figure 2). The occurrence of nausea (24 patients in the $\mathrm{M}$ group versus 48 in the $\mathrm{C}$ group, $\mathrm{p}=0.005$ ) and vomiting (4 patients in the $M$ group versus 68 in the $C$ group, $p=0.001$ ) was significantly lower in the $M$ group. The total administered dosage of ephedrine was significantly lower in the $\mathrm{M}$ group (median $4.1 \mathrm{mg} \mathrm{vs}$. 17.1 $\mathrm{mg}, \mathrm{p}=0.001$ ). There was no difference in neonatal Apgar scores at 1 and 5 minutes between groups.

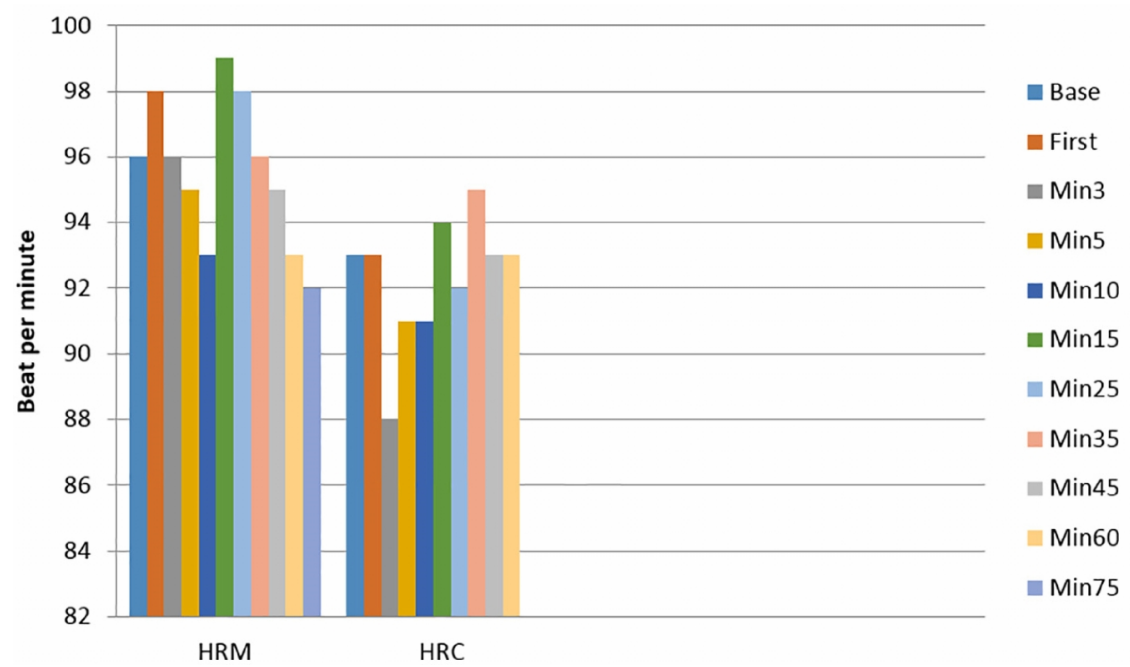

Figure 2. Serial changes in heart rate. Data are presented as mean $\pm \mathrm{SD}$. Heart rate changes were significantly higher in $\mathrm{M}$ group in the first and third minutes $(\mathrm{p}<0.05)$. HR: Heart Rate. $\mathrm{M}$ and $\mathrm{C}$ denote treatment mechanical pump group and control group respectively.

Table1. Patient characteristics and surgical times

\begin{tabular}{|l|l|l|l|}
\hline Variables & Group M (n=35) & Group C $(\mathrm{n}=38)$ & $p$-value \\
\hline Age (yr.) & $30(1.65)$ & $29.5(2.1)$ & 0.65 \\
\hline Weight (kg) & $65(7.8)$ & $69(8.5)$ & 0.10 \\
\hline Height (cm) & $155(4.8)$ & $154(5.1)$ & 0.13 \\
\hline Block height (dermatome) & T5(T4-T6) & T4(T4-T6) & 0.94 \\
\hline Induction to delivery time (min) & $25(23.5-27.5)$ & $26(24.3-28.2)$ & 0.89 \\
\hline Incision to delivery time (min) & $7.8(6.5-12)$ & $8.2(6.8-10.2)$ & 0.75 \\
\hline Uterine incision to delivery time(s) & $75(60-85)$ & $82(58-102)$ & 0.60 \\
\hline
\end{tabular}

Values are mean \pm standard deviation or median

Table 2. Hemodynamic changes and Ephedrine requirement

\begin{tabular}{|l|l|l|l|}
\hline Variables & Group M $(\mathrm{n}=35)$ & Group C $(\mathrm{n}=38)$ & $p$-value \\
\hline Total ephedrine dose (mg) & $4.1(3-5.5)$ & $17.1(13.8-20.4)$ & 0.001 \\
\hline Incidence of hypotension & 1 & 3 & 0.75 \\
\hline Minimum recorded SBP (mmhg) & $112(122-112)$ & $109(135-109)$ & 0.06 \\
\hline Incidence of hypertension & $0(\% 0)$ & $0(\%)$ & 1 \\
\hline Maximum recorded SBP (mmhg) & $122(112-122)$ & $135(109-135)$ & 0.51 \\
\hline Minimum recorded DBP (mmhg) & $67(75-67)$ & $61(85-61)$ & 0.87 \\
\hline Maximum recorded DBP (mmhg) & $75(67-75)$ & $85(61-85)$ & 0.80 \\
\hline Minimum recorded MBP (mmhg) & $81(81-92)$ & $77(77-93)$ & 0.60 \\
\hline Maximum recorded MBP (mmhg) & $92(92-81)$ & $93(93-77)$ & 0.90 \\
\hline Minimum recorded of HR (beats/min) & $92(99-92)$ & $88(95-88)$ & 0.90 \\
\hline Atropine required & 0 & 0 & 1 \\
\hline Incidence of bradycardia (HR $<50$ beats/min) & 0 & 0 & 1 \\
\hline
\end{tabular}

Values are median or number $(\%) ; \mathrm{HR}=($ Heart Rate $) ; \mathrm{SBP}=($ Systolic Blood Pressure $) ; \mathrm{DBP}=($ Diastolic Blood Pressure) 


\section{Discussion}

According to the findings section, hypotension in the pneumatic compression group or M group is not significantly different from the control group, but the administrated dosage of ephedrine was significantly lower in the pneumatic compression group. In addition, the incidence of nausea and vomiting was considerably lower in the pneumatic group and literature review produced almost similar results. For example, Sujata randomly divided 100 pregnant women, undergoing elective C-section under spinal anesthesia, in pneumatic compression and control groups. The protocols of standard pre-surgery fluid therapy and spinal anesthesia were similar in both groups. Hypotension was defined by a systolic pressure drop by more than $20 \%$ of the baseline and was cured with 6 mg intravenous bolus ephedrine. The incidence of hypotension was considered as the primary outcome and the median dosage of the administered ephedrine was determined. The hypotension rates were $25.5 \%$ and $60 \%$ in the treatment and control groups, respectively ( $\mathrm{p}=0.001)$. This was inconsistent with the findings of our study; however, the median ephedrine dosages in their study were 12 [0-24] $\mathrm{mg}$ in the treatment group and 0 [0-12] $\mathrm{mg}$ in the control group ( $\mathrm{p}<0.001)$. On the other hand, there was no difference between groups in the time to the onset of hypotension (9), which was almost consistent with our findings. In addition, Adsumelli randomly divided 50 pregnant women, undergoing Csection into two groups of SCD and control, each with 25 subjects. Similarly, a standard fluid therapy method and spinal anesthesia was applied to all patients. Hypotension was defined by a systolic pressure drop by more than $20 \%$ of the baseline. The baseline indices of systolic pressure, average blood pressure, diastolic blood pressure, pulse pressure, and heart rate were recorded after spinal block, every minute until the delivery of newborn. Median hypotension drop was observed in $52 \%$ of the SCD and $92 \%$ of the control groups ( $\mathrm{p}=0.004)$. There was no significant difference between the two groups in terms of systolic pressure, diastolic pressure, heart rate, and pulse pressure (1). This finding agrees with ours, as the difference was significant only in a few minutes. Bishnu et al. divided 100 pregnant women, undergoing C-section under spinal anesthesia, into SCD and control groups. They then compared the two groups in terms of the rate and duration of hypotension drop, mean dosage of required ephedrine, average amount of blood loss, and sensory block level. In 24\% of SCD and 54\% of control groups, hypotension drop was significant $(\mathrm{p}=0.002)$. The mean dosages of administered ephedrine were $10 \mathrm{mg}$ and $3.15 \mathrm{mg}$ in SCD and control groups, respectively $(\mathrm{p}=0.008)$. There was no difference between groups in the time to the onset of hypotension drop. The average blood loss and sensory block level were comparable between the two groups (5).

\section{Conclusions}

This research showed the suitability of the use of Sequential Compression Device (SCD) in reducing hypotension after spinal anesthesia for cesarean section, also this method can cause reducing vasopressor dosage for increased blood pressure, but the approval of its effectiveness requires repetition of the study with a larger sample size.

\section{Acknowledgments:}

This research was approved by the Pain Research Center, Ahvaz Jundishapur University of Medical Sciences, Ahvaz, Iran (Proposal no. PAIN-9312). The authors appreciate the assistance and of the women who participated in the study. Also, we appreciate the assistance consultation by Dr. M.R. Pipelzadeh (Ahvaz Jundishapur University of Medical Sciences, Ahvaz, Iran) and Ms. Sara Adarvishy who was collector of data (Ahvaz Jundishapur University of Medical Sciences, Ahvaz, Iran).

\section{Trial Registration:}

The trial was registered at the Iranian Registry of Clinical Trials (http://www.irct.ir) with the Irct ID: IRCT2015011217742N3.

\section{Funding:}

The authors received no financial support for the research, authorship, and/or publication of this article.

\section{Conflict of Interest:}

There is no conflict of interest to be declared.

\section{Authors' contributions:}

All authors contributed to this project and article equally. All authors read and approved the final manuscript. 


\section{References:}

1) Adsumelli RS, Steinberg ES, Schabel JE, Saunders TA, Poppers PJ. Sequential compression device with thigh - high sleeves supports mean arterial pressure during Caesarean section under spinal anaesthesia. Br J Anaesth. 2003; 91(5): 695-8. doi: 10.1093/bja/aeg248. PMID: 14570793.

2) Grant GJ, Hepner DL, Barss VA. Anesthesia for cesarean delivery. Retreived on April. 2011; $28: 2011$.

3) Mitra JK, Roy J, Bhattacharyya P, Yunus M, Lyngdoh NM. Changing trends in the management of hypotension following spinal anesthesia in cesarean section. J Postgrad Med. 2013; 59(2): 121-6. doi: 10.4103/0022-3859.113840. PMID: 23793313.

4) American Society of Anesthesiologists Task Force on Obstetric Anesthesia. Practice guidelines for obstetric anesthesia: an updated report by the American Society of Anesthesiologists Task Force on Obstetric Anesthesia. Anesthesiology. 2007; 106(4): 843-63. doi: 10.1097/01.anes.0000264744.63275.10. PMID: 17413923.

5) Panigrahi BP, Nambiath S, Arora D, Das S. Use of a Sequential Compression Mechanical Device to Prevent Spinal Hypotension in Elective Cesarean Section. Anesthesiology. 2011.

6) Mercier FJ, Augè M, Hoffmann C, Fischer C, Le Gouez A. Maternal hypotension during spinal anesthesia for caesarean delivery. Minerva Anestesiol. 2013; 79(1): 62-73. PMID: 23135692.

7) Norris MC. Hypotension During Spinal Anesthesia for Cesarean Section: Does it Affect Neonatal Outcome? Regional Anesthesia and Pain Medicine. 1987; 12(4): 191 -4.

8) Reynolds F, Seed PT. Anaesthesia for Caesarean section and neonatal acid - base status: a meta - analysis. Anaesthesia. 2005; 60(7): 636-53. doi: 10.1111/j.1365-2044.2005.04223.x. PMID: 15960713.

9) Sujata N, Arora D, Panigrahi BP, Hanjoora VM. A sequential compression mechanical pump to prevent hypotension during elective cesarean section under spinal anesthesia. Int J Obstet Anesth. 2012; 21(2): 1405. doi: 10.1016/j.ijoa.2012.01.003. PMID: 22398360. 